\title{
Cross-cultural adaptation and measurement properties of the Dutch knee self efficacy scale (K-SES)
}

Wim van Lankveld ${ }^{1 *}$ (D) Nicky van Melick ${ }^{2,3}$, Bas Habets ${ }^{4,5}$, Yvette Pronk ${ }^{6}$, J. Bart Staal ${ }^{1,2}$ and Robert van Cingel ${ }^{2,4}$

\begin{abstract}
Background: Self-efficacy is related to outcome after anterior cruciate ligament (ACL) tears. The Knee Self Efficacy Scale (K-SES) available in Swedish and English, was developed to measure self-efficacy in present (K-SES present) and future (K-SESfuture) functioning. The objective of this study was to determine measurement properties of the K-SES in Dutch patients.
\end{abstract}

Methods: The K-SES was translated and structural validity, internal consistency, test-retest reliability, and measurement error were assessed in three patient samples: one group completed the questionnaire and additional measures pre-surgery $(N=200)$, and one group post-surgery $(N=58)$. The third group (post-surgery) completed the K-SES twice $(N=50)$.

Results: Exploratory factor analysis distinguished two underlying important factors: K-SESpresent and K-SES future. However, the distinction was not confirmed in Confirmatory Factor Analysis (CFA). Internal consistency for both subscales was excellent (Cronbach's alpha $>$.80). Test-retest reliability absolute agreement was 0.95 . A-priori formulated hypotheses on the relation between Knee Self Efficacy Scale Dutch (K-SES-D) and related constructs were confirmed. Moderate to high correlations $(r>0.50)$ were reported with Knee Injury and Osteoarthritis Outcome Score (KOOS) before reconstruction. High negative correlation was found with fear of movement and pain catastrophizing $(r<-0.60)$, and low correlation $(r<0.50)$ with locus of control and measures of distress.

Conclusion: Acceptability, internal consistency and test-retest reliability of the K-SES-D subscales are satisfactory. Construct validity of both subscales was confirmed by exploratory factor analysis and hypothesis testing. However, construct validity was not confirmed in CFA. Further research is needed to test responsiveness.

Keywords: Anterior cruciate ligament, Reconstruction, Self-efficacy

\section{Background}

An anterior cruciate ligament (ACL) tear is a frequently reported injury in sports activities that include pivoting movements of the knees, such as soccer, basketball, football, handball, and skiing. The incidence of ACL tears is estimated at 68.6 per 100,000 person years [1]. An ACL tear is a painful condition and has a large impact on a patient's functioning [2]. Both conservative and reconstructive (ACLR) treatment options are available [3]. However, most athletes in pivoting sports will opt for an

\footnotetext{
* Correspondence: Wim.vanlankveld@han.nl

'Research group Musculoskeletal Rehabilitation Nijmegen, HAN University of Applied Sciences, Kapittelweg, 33 Nijmegen, The Netherlands

Full list of author information is available at the end of the article
}

ACLR procedure. Rehabilitation after ACLR involves exercises and training to enhance the athlete's performance in sports activities [4]. Despite extensive rehabilitation, it is estimated that only $50-65 \%$ of the patients with ACLR return to their pre-injury level of sports $[5,6]$.

Psychological factors are important predictors of return to sport in injured athletes in general [7], and this also applies to ACL injured athletes $[8,9]$. Psychological variables related to return to sport in ACLR include catastrophizing cognitions and kinesophobia [10], levels of stress [11], and self-efficacy beliefs [12]. Self-efficacy beliefs also predict outcome after ACL tears [13]. A recent systematic review showed strong support for the role of self-efficacy in predicting ACLR outcome [9]. As these psychological variables

(c) The Author(s). 2019 Open Access This article is distributed under the terms of the Creative Commons Attribution 4.0 International License (http://creativecommons.org/licenses/by/4.0/), which permits unrestricted use, distribution, and reproduction in any medium, provided you give appropriate credit to the original author(s) and the source, provide a link to the Creative Commons license, and indicate if changes were made. The Creative Commons Public Domain Dedication waiver (http://creativecommons.org/publicdomain/zero/1.0/) applies to the data made available in this article, unless otherwise stated. 
are relevant for rehabilitation outcome in ACL patients, they should be assessed and addressed in ACL rehabilitation [14].

To be able to identify self-efficacy in ACL and ACLR patients, researchers have used different instruments [9]. Bandura suggested that self-efficacy should be measured as specific as possible by measuring self-efficacy beliefs related to the target behaviour [15]. In line with these recommendations, the Knee Self-Efficacy Scale (K-SES) was developed in Sweden to assess self-efficacy beliefs related to behaviour after ACL tears and is previously published in Swedish and English [16]. The K-SES consists of 22 items measuring self-efficacy in daily activities, sports and leisure activities, physical activity, and knee function in the future. Based on dimension reduction techniques, two important factors were identified [16]. The first factor refers to self-efficacy related to present functioning (K-SES present $)$ measured with items related to daily activities, sports and leisure activities, and physical activities. The second factor measures knee function in the future $\left(\mathrm{K}-\mathrm{SES} \mathrm{S}_{\text {future }}\right)$. The K-SES has shown good internal validity, face validity, and convergent validity. In a longitudinal study, it was shown that self-efficacy assessed with the K-SES prior to surgery was a predictor of ACLR outcome 1 year after surgery [13].

However, the K-SES is not available in Dutch, and no data have been reported on the goodness of fit of the two factor model in a confirmatory factor analysis. Therefore, the aim of this study was 1) to cross-culturally adapt the K-SES into Dutch according to international accepted guidelines, and 2) to determine measurement properties of the K-SES Dutch language version (K-SES-D) including goodness of fit for the two factors.

\section{Methods}

\section{Cross cultural adaptation of the K-SES}

A cross cultural adaptation of the K-SES into Dutch was performed according to international accepted guidelines [17]. The K-SES items asses self-efficacy in the domains of daily activities (7 items); sports activities (5 items); physical function tasks (6 items); and knee function in the future ( 4 items). In a factor analysis the 22 items reflect two dimensions: self-efficacy beliefs in current functioning (K-SES present ) and self-efficacy towards future functioning $\left(\mathrm{K}-\mathrm{SES}_{\text {future }}\right)$. Items are scored on an 11-point scale $(0=$ not certain at all and $10=$ very certain). The Beaton method involves different steps of forward and backward translation using two independent translators for each step. Consensus discussions between the independent translators were used to resolve any discrepancies between translation. Acceptability of the translated Dutch version was checked in a small sample of 15 ACLR patients using structured interviews by phone. Patients were asked if they had any difficulty completing the questionnaire, and whether they had suggestions about any item.

\section{Measurement properties}

The Consensus-based Standards for the selection of health Measurement Instruments (COSMIN) [18] was used as a guide in determining measurement properties of the K-SES-D. The following COSMIN measurement properties will be described: structural validity, internal consistency, reliability, measurement error, and construct validity using hypothesis testing.

\section{Patient samples}

The final version of the K-SES-D was used in three different samples of ACL patients. The first sample consisted of 200 patients of Kliniek ViaSana (Mill, the Netherlands) with an ACL tear scheduled for ACLR with quadruple semitendinosus graft. All patients completed the K-SES-D on the computer, prior to reconstructive surgery, as part of a larger clinical prospective cohort study. Inclusion criteria for this study were: age between 16 and 50 years, Tegner Activity Scale $\geq 6$, undergoing ACLR. Excluded were patients undergoing revision surgery, or patients with an ACL tear of the contralateral knee in the past.

The second sample consisted of 72 ACLR patients, not included in sample 1, who were invited to participate in a study conducted in 2015 to develop the Photographic Series of Sports Activities for Anterior Cruciate Ligament Reconstruction (PHOSA-ACLR), a new instrument to measure fear of harm or re-injury after ACLR [19]. Patients in sample 2 were included in this study between 3 and 36 months after reconstruction. In 2016, a third sample of 50 ACLR rehabilitation patients completed the K-SES-D on paper twice with an interval of 1 week. Additional file 1: Figure S1 shows details about the 3 independent samples in the study and the purpose they were used for.

Patients in sample 2 and 3 with an ACLR were invited through direct referral by two physical therapists working in Sports Medical Centres (Sports Medical Center Papendal, Arnhem, the Netherlands; and Funqtio, Steyl, the Netherlands), or through referral from primary care physical therapists in the Nijmegen area associated with the HAN University of Applied Sciences. Patients older than 16 years of age were asked to participate in this study if they had undergone ACLR. Exclusion criteria were: unable to read or write Dutch, and co-morbidity affecting knee function. Questionnaires were filled in during a visit to the physical therapist using a hardcopy query. When data were incomplete the patient was asked to complete the questionnaire.

\section{Measurements}

All participants reported demographics, and time since ACLR. Based on the K-SES-D scores, average item scores 
were computed for K-SES-D present (18 items), K-SES$\mathrm{D}_{\text {future }}$ ( 4 items), and K-SES-D total $(22$ items). To be able to compare samples, knee related function was assessed in samples 1 and 2 using the validated Dutch version of the Knee Injury and Osteoarthritis Outcome Score (KOOS) [20]. The KOOS measures pain, other disease-specific symptoms, activities of daily life (ADL) function, sport and recreation function, and knee-related quality of life (QOL). For each scale the scores were recoded from 0 to 100 , with 100 depicting no problems.

To determine structural validity of the K-SES-D, sample 2 completed a set of additional questionnaires. Fear of movement was assessed using the Dutch version of the Tampa Scale of Kinesiophobia (TSK) [21]. Catastrophizing cognitions regarding pain were measured using the validated Dutch version of the Pain Catastrophizing Scale (PCS) [22]. Health Locus of Control was assessed using the validated Dutch version of the Multidimensional Health Locus of Control Scale (MHLC) [23], measuring internal, external, and physician locus of control. Finally, distress was measured using the validated Dutch version of the Hospital Anxiety and Depression Scale (HADS) [24]. For each scale in the study the validated Dutch version was used, and a higher scores means that the patients reports higher levels of the assessed construct.

Data of sample 1 and 2 were sampled using a web based program, and patients had to complete every question. Therefore, there are no missing individual items in these samples. Sample 3 completed a paper version of the questionnaire, and data completeness was checked when patients handed in the questionnaires. When data were incomplete the patient was asked to complete the questionnaire.

\section{Data analysis}

Metric data were described using means, and standard deviations (SDs). Item descriptives and reliability were studied in sample 1 and 2 in a similar way as described by Thomeé et al. [16]. Measures of Skewness between 2 and +2 are considered acceptable for normal univariate distribution. The strength of pearson correlations between variables is defined as negligible (.00 to .30 ), low (.30 to .50$)$, moderate (.50 to .70$)$, high (.70 to .90$)$, and very high (.90 to 1.00 , [25]). Differences between independent groups were tested using the $\mathrm{T}$ statistic, and significance of the difference is reported. Regression analysis was used to determine the multiple correlation (R) between a set of independent variables and the subscales of the K-SES-D. For these multiple regressions, both multiple correlation $(\mathrm{R})$, and percentage of variation explained $\left(R^{2}\right)$ were reported.

Structural validity was investigated using Principal Component Analysis (PCA). As the structure of the original K-SES reflects two important factors of self-efficacy.
The first factor refers to self-efficacy related to present functioning (K-SES $\mathrm{present})$ with items related to daily activities, sports and leisure activities, and physical activities. The second factor measures self-efficacy beliefs related to knee function in the future $(\mathrm{K}-\mathrm{SES}$ future). [16], it is expected that the same two important factors will be observed in this study. Therefore, the underlying factor structure of the K-SES-D in combined data from sample 1 and 2 was analysed using PCA [26]. First, sampling adequacy was determined using Kaiser-Meyer-Olkin (KMO) and Bartlet test. Sampling adequacy is considered good when $\mathrm{KMO}$ value $>.8$, and Bartlett test of sphericity is significant. Next, a maximum likelihood PCA with Harris Kaiser's rotation in a two factor solution was conducted. It was expected that the items related to present physical performance would have significant loadings on the first factor, and that the items of the scale knee function in the future would have significant loadings on the second factor. In addition, a Confirmatory Factor Analysis (CFA) was used to test whether the observed item scores of the K-SES-D in sample 1 and 2 fit the two factor model. In CFA different indicators are used to depict goodness of fit [27]. In this study four indices of absolute fit were used: Chi-squared test corrected for degrees of freedom (CMIN/DF), the Root Mean square error of estimation (RMSEA), the Comparative fit index (CFI), and the standardised root mean square residual (SRMR). Acceptable fit is indicated by CMIN/DFD < 5.0, RMSA < 0.6, CFI > 0.90 , and SRMR $<0.08$ [27]. Three measurement properties of reliability were assessed for this study: internal consistency, test-retest reliability, and measurement error [18]. Internal consistency was calculated using Cronbach Alpha. A value of Cronbach Alpha $>.80$ is considered excellent [28]. Cronbach alpha was calculated separately for K-SES-D present,$K-S E S-D_{\text {future }}$, and K-SES- $D_{\text {total }}$ in samples 1 and 2. Test-retest reliability was calculated based on two independent assessments of the instrument in sample 3 . Mean differences between test and re-test item scores were calculated with corresponding $95 \%$ confidence interval (CI). When zero lies within the $95 \% \mathrm{CI}$ this is considered a criterion for absolute agreement. Finally, for each K-SES-D scale intraclass correlation coefficients (ICC) between both assessments were calculated with corresponding $95 \% \mathrm{CI}$ to determine absolute agreement between assessments. The two way random effects model was used. An ICC above 0.75 is considered good [29]. Standard error of measurement (SEM) was computed in the same sample by dividing the $\mathrm{SD}$ of the mean difference between both assessments (SDdiff) by $\sqrt{ } 2$ [29].

Construct validity was further investigated by determining the degree to which the scores on the instrument are consistent with a-priori defined hypotheses. Table 1 shows the hypotheses that have been formulated based on the reported construct validity of the original manuscript. It 
Table 1 Hypotheses about the relation between K-SES-D and other variables

\begin{tabular}{|c|c|}
\hline 1 & $\begin{array}{l}\text { K-SES-D will show } 2 \text { dimensions in exploratory factor analysis: } \\
\text { present and future self-efficacy. }\end{array}$ \\
\hline 2 & $\begin{array}{l}\text { K-SES-D will fit the proposed } 2 \text { dimension model using } \\
\text { confirmatory factor analysis. }\end{array}$ \\
\hline 2 & $\begin{array}{l}\text { K-SES- } D_{\text {present }} \text { will have a high positive correlation with knee } \\
\text { function (KOOS) }(0.50<r<0.80) \text {. }\end{array}$ \\
\hline 3 & $\begin{array}{l}\text { K-SES- } D_{\text {future }} \text { will have a low correlation with knee function } \\
\text { (KOOS) }(0.30<r<0.50) \text {. }\end{array}$ \\
\hline $4-5$ & $\begin{array}{l}\text { K-SES- } D_{\text {present }} \text { and K-SES- } D_{\text {future, }} \text { both have moderate to high } \\
\text { correlations with TSK }(0.50<r<0.80) \text {. }\end{array}$ \\
\hline $6-7$ & $\begin{array}{l}\text { K-SES-D present, } K-S E S-D_{\text {future }} \text { both will have strong correlations } \\
\text { with PCS }(0.50<r<0.80) \text {. }\end{array}$ \\
\hline 8 & $\begin{array}{l}\text { K-SES-D present, } K-S E S-D_{\text {future, }} \text {, both will have low association } \\
\text { with } M H L C(0.30<r<0.50) \text {. }\end{array}$ \\
\hline 9 & $\begin{array}{l}\text { K-SES-D present, K-SES-D } D_{\text {future, }} \text { both will have low association } \\
\text { with HADS }(0.30<r<0.50) \text {. }\end{array}$ \\
\hline
\end{tabular}

Abbrevations: K-SES- $D_{\text {present }}$ Knee Self-Efficacy Scale present function, $K$-SES$D_{\text {future }}$ Knee Self-Efficacy Scale present function, KOOS Knee Injury and Osteoarthritis Outcome Score, TSK Tampa Scale of Kinesiophobia, PCS Pain Catastophizing Scale, MHLC Multidimensional Health Locus of Control, HADS Hospital Depression and Anxiety Scale

was expected that exploratory factor analysis would result in 2 important factors: K-SES-D $D_{\text {present }}$ and K-SES-D future. K-SES-D $\mathrm{D}_{\text {present }}$ was expected to be highly correlated with self-reported knee functioning, whereas lower correlations

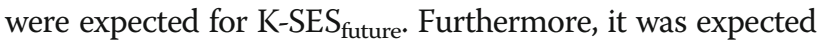
that $\mathrm{KSES}_{\text {present }}$, and K-SES future, would have moderate to high reversed correlations with levels of both fear of movement, and catastrophizing, and low to moderate correlations with locus of control and distress. The construct validity is good when $>75 \%$ of the a-priori formulated hypotheses about the relation of the construct with other theoretically derived constructs are confirmed [29].

\section{Results}

Consensus was reached in the translation panel with only minor differences resolved. The final Dutch version was endorsed by 15 patients receiving physical therapy treatment after an ACLR (9 males (60\%), mean age is 21 years (S.D. $=2.8)$, average number of months since reconstruction is 4 (S.D. $=5.6)$. The back-translation was endorsed by the author of the original K-SES study (personal communication R. van Cingel).

Table 2 displays the demographic characteristics of both sample 1 and 2 and the scores for self-reported knee function scales.

Average number of months since ACL for patient on the waiting list in sample 1 was 6.7 months (S.D. $=11.1)$. In sample 2, $58(77 \%)$ of the 75 eligible patients participated. Time since reconstruction is 12.9 month (range = $2-48)$. The proportion of males was higher in the ACL group awaiting surgery $(\mathrm{Z}$ score for difference between two independent proportions $\mathrm{Z}=3.6, p<.001)$. The ACL
Table 2 Characteristics of patients in the ACL tear group presurgery (sample 1: ACL) and in rehabilitation after reconstruction surgery (sample 2: ACLR), and T value for independent samples

\begin{tabular}{llll}
\hline & $\begin{array}{lll}\text { ACL } \\
(N=200)\end{array}$ & $\begin{array}{l}\text { ACLR } \\
(N=58)\end{array}$ & T. \\
\hline Age (mean; SD) & $23.7(8.5)$ & $25.9(8.2)$ & n.s. \\
Gender: \% male & $69.0 \%$ & $43.0 \%$ & \\
KOOS pain (0-100) (mean; SD) & $70.0(18.2)$ & $81.8(17.7)$ & $4.3^{* *}$ \\
KOOS symptoms (0-100) (mean; SD) & $63.5(20.7)$ & $63.2(12.8)$ & n.s. \\
KOOS ADL (0-100) (mean; SD) & $82.0(17.7)$ & $89.1(14.2)$ & $2.8^{* *}$ \\
KOOS Sports and Leisure (0-100) & $37.7(26.6)$ & $59.1(30.6)$ & $5.2^{* *}$ \\
(mean; SD) & & & \\
KOOS QOL (0-100) (mean; SD) & $38.7(16.7)$ & $48.3(14.1)$ & $3.9^{* *}$ \\
\hline
\end{tabular}

Abbrevations: $A C L$ Anterior Cruciate Ligament, $A C L R$ Anterior Cruciate Ligament Reconstruction, $N$ number of participants, n.s. not significant, KOOS Knee Injury and Osteoarthritis Outcome Score, ADL Activities of Daily Life, QOL Quality of Life

${ }^{* *} p<.001$

group awaiting surgery reported poorer scores in KOOS pain, ADL, Sports and Leisure, and QOL $(p<.001)$ when compared to the ACLR group (sample 2). The difference in KOOS subscale scores may be expected as the average time since ACL between both groups is very different. Item characteristics of the K-SES-D items of sample 1 and 2 were analysed first. Item scores ranged from 0 to 10 for each item. All items showed symmetry of frequency distribution, with measured of Skewness for all items $<|1|$.

\section{Structural validity}

Factor analysis in the combined data from sample 1 and 2 was adequate $(\mathrm{KMO}=0.95$, and Bartlet test significant < .001). PCA using Harris Kaiser's factor rotation resulted in 2 important factors. Items of the K-SES-D reflecting present physical performance/function had high loading on the first factor (all factor loadings $>0.70$ ). This factor K-SES- $D_{\text {present }}$ explained $56 \%$ of the variance of the K-SES-D total. The second factor (K-SES- $\mathrm{D}_{\text {future }}$ ) explained an additional $12 \%$ of the variation in item scores with only the four items of the knee function in the future subscale demonstrating high loadings. In a confirmatory factor analysis the two factor model could not be confirmed. Only two of the four indices indicated acceptable fit of the model $(\mathrm{SRMR}=0.05 ; \mathrm{CMIN} / \mathrm{DF}=4.9 ; \mathrm{CFI}=$ 0.85 ; RMSEA $=0.12$ ). Inspection of the covariance matrix showed that in particular the 18 items relating to K-SES-D $D_{\text {present }}$ did not fit well into the CFA.

\section{Reliability}

Average scale scores were calculated for K-SES-D present and K-SES- $\mathrm{D}_{\text {future }}$ and K-SES-D $\mathrm{D}_{\text {total }}$ score, both in sample 1 (ACL), and 2 (ACLR). In Table 3 scale statistics are given for both subscales of the K-SES and total score for both samples. 
Table 3 Descriptive data on final K-SES-D from two independent samples: sample 1 (ACL patients; $N=200$ ), and sample 2 (ACLR patients; $N=58$ )

\begin{tabular}{|c|c|c|c|c|c|c|}
\hline & \multicolumn{2}{|c|}{ K-SES-D present } & \multicolumn{2}{|c|}{ K-SES-D $D_{\text {future }}$} & \multicolumn{2}{|c|}{ K-SES-D total } \\
\hline & $\overline{\mathrm{ACL}}$ & $\overline{A C L R}$ & $\overline{\mathrm{ACL}}$ & $\overline{A C L R}$ & $\overline{\mathrm{ACL}}$ & ACLR \\
\hline$\overline{M e a n}$ & 4.6 & 7.9 & 6.2 & 5.3 & 5.4 & 7.2 \\
\hline SD & 2.3 & 2.0 & 2.1 & 2.6 & 1.7 & 1.9 \\
\hline Cronbach's Alpha & 0.92 & 0.91 & 0.83 & 0.81 & 0.95 & 0.96 \\
\hline 95\% Cl lower & 4.3 & 7.4 & 5.9 & 4.6 & 4.7 & 6.7 \\
\hline 95\% Cl upper & 4.9 & 8.4 & 6.5 & 6.0 & 5.3 & 7.7 \\
\hline Observed range & $0-10$ & $0-10$ & $0-10$ & $0-10$ & $0-10$ & $0-10$ \\
\hline Skewness & 0.15 & -1.1 & -0.56 & -0.25 & 0.12 & -0.98 \\
\hline
\end{tabular}

Abbrevations: K-SES-D Knee Self Efficacy Scales Dutch, $A C L$ Anterior Cruciate Ligament, $A C L R$ Anterior Cruciate Ligament Reconstruction, $S D$ Standard Deviation, $\mathrm{Cl}$ confidence interval

Cronbach's Alpha as a measure of internal consistency for each subscale is good, and measures of skewness indicate normal distribution. K-SES- $\mathrm{D}_{\text {present }}$ in the ACL group was significantly lower compared to mean score in the ACLR group $(\mathrm{T}=9.9, p<.0001)$. With regard to K-SES- $\mathrm{D}_{\text {future }}$ the relation was reversed: ACL patients reported higher levels of efficacy beliefs with regards to their future knee functioning compared to ACLR patients $(\mathrm{T}=2.71, \mathrm{df}=256 ; p<.001)$.

Reliability or test-retest reliability for the K-SES- $\mathrm{D}_{\text {present }}$ and K-SES- $D_{\text {future }}$ subscales was computed in sample 3 consisting of 50 ACLR patients. Average age in this sample was 25.8 years (range 16-51), with 57\% males, and time since reconstruction was on average 6 months (range $2-21$ ). Average change score for K-SES- $D_{\text {present }}$ was -0.36 (95\% CI $-0.62,-0.09)$, with $\mathrm{SD}=0.92$. Test-retest ICC between both assessments was $0.93(95 \%$ CI $0.88,0.96)$ and SEM was 0.13 . Average change score for K-SES-D future $_{\text {fur }}$ was -0.14 (95\% CI $-0.35,0.08)$, with $\mathrm{SD}=0.74$. Test-retest ICC between both assessments was 0.92 (95\% CI $0.86,0.95)$ and SEM was 0.46.

\section{Construct validity (using hypothesis)}

Correlations were computed between measures of self-efficacy on the one hand, and knee function variables in sample 1 and 2 on the other hand. Table 4 shows the correlations between K-SES-D subscales on the one hand, and self-reported knee functioning assessed with the KOOS subscales on the other. For both K-SES subscales multiple correlation $(R)$, and percentage of variation explained $\left(R^{2}\right)$ are given depicting the strength of the relation between the combined set of KOOS scales with K-SES-D scales.

With the exception of the KOOS symptom subscale, all correlations between KOOS subscales and K-SES- $\mathrm{D}_{\text {present }}$ scores were moderate to high.

In Table 5, correlations are given between K-SES-D $\mathrm{D}_{\text {present }}$, K-SES- $\mathrm{D}_{\text {future, }}$ and K-SES- $\mathrm{D}_{\text {total }}$ scores on the one hand, and the psychological variables on the other hand in sample 1. Psychological variables include fear of movement, pain catastrophizing, health locus of control, and anxiety and depression.

K-SES-D scale scores had moderate to high $(r>|.50|)$ negative correlations with TSK, and PCS. Negligible to low correlations were found between with MHLC scale scores, and HADS score. Together, these variables explained 55,51 , and $63 \%$ of the variation in the K-SES scales. Three stepwise regression analyses were performed with K-SES-D $D_{\text {present }}$, K-SES-D future, and K-SES-D $_{\text {total }}$ scores as dependent variables. Self-reported knee function assessed using the KOOS subscales explained $74 \%$ of the variance in K-SES- $D_{\text {present }}$. Entering the combined psychological variables explained an additional $7 \%$ of the variation in K-SES-D $D_{\text {present }}(\mathrm{F}$ change $=2.4 ; \mathrm{df}=6,46 ; p<.05)$, $21 \%$ of variation K-SES- $\mathrm{D}_{\text {future }}$, and $7 \%$ of the variation in K-SES-D total $_{\text {tal }}$ (F change $=3.2 ; \mathrm{df}=5,47 ; p<.05$ ).

All except one of the hypotheses formulated in Table 1 were confirmed in the analysis. Hypotheses 2 was rejected as the FCA did not confirm a 2 factor model underlying the data.

\section{Discussion}

Measurement properties of the translated K-SES-D were good, with good reliability and validity. Exploratory factor analysis revealed two factors underlying the scores on the K-SES-D similar to the factors reported in the original study: self-efficacy beliefs about daily activities, sports and leisure activities, and physical activity (K-SES- $\mathrm{D}_{\text {present }}$ ), and self-efficacy beliefs about knee function in the future (K-SES- $\left.\mathrm{D}_{\text {future }}\right)$. However, in a CFA, this hypothesised 2 -factoral model could not be confirmed. More than $75 \%$ of a-priori formulated hypotheses were confirmed.

The negligible to low correlation with Internal Health Locus of Control underlines the importance of measuring self-efficacy beliefs as specific as possible related to the target behaviour [15]. As rehabilitation after ACLR involves exercises and training to enhance the patient's performance in sports activities [30], there is a need to understand the athlete's beliefs regarding exercise and training. The K-SES assesses these beliefs [16], but item responses did not fit the hypothesised two factor model in a CFA. This means that the items of this scale are not consistent with a researcher's understanding of the nature of that construct (or factor) [29]. In particular, the items on the K-SES- $\mathrm{D}_{\text {present }}$ factor do not fit well in the model. These findings are consistent with the way the instrument was developed. The authors generated items to be categorized in four a-priori defined groups: daily activities, sports and leisure activities, physical activities, and knee functioning in the future [16]. In an exploratory factor analysis of the original data, which was replicated in this study, two important factors emerged, $\mathrm{K}-\mathrm{SES}$ present, and $\mathrm{K}_{-} \mathrm{SES} \mathrm{S}_{\text {future. }}$ Items 
Table 4 Pearson correlation (95\% Confidence interval) between K-SES-D and KOOS self-report knee function scales in sample 1 $(A C L)$ and $2(A C L R)$

\begin{tabular}{|c|c|c|c|c|c|c|}
\hline \multirow[b]{2}{*}{ KOOS } & \multicolumn{2}{|c|}{ K-SES-D present } & \multicolumn{2}{|l|}{ K-SES- $D_{\text {future }}$} & \multicolumn{2}{|l|}{$\mathrm{K}-\mathrm{SES}-\mathrm{D}_{\text {total }}$} \\
\hline & $\overline{\mathrm{ACL}}$ & ACLR & $\overline{\mathrm{ACL}}$ & ACLR & $\mathrm{ACL}$ & ACLR \\
\hline Pain & $.64(.53, .73)$ & $.82(.89, .67)$ & $.16(.00, .31)$ & $.54(.33, .72)$ & $.61(.48, .72)$ & $.82(.68, .89)$ \\
\hline Sympt & $.44(.30, .55)$ & $.27(.05, .59)$ & $.15(.00, .32)$ & $.37(.10, .59)$ & $.43(31,56)$ & $.34(.00, .64)$ \\
\hline $\mathrm{ADL}$ & $.63(.51, .72)$ & $.82(.64, .90)$ & $.16(.01, .33)$ & $.46(.24, .67)$ & $.60(.47, .71)$ & $.79(.61,69)$ \\
\hline Sport & $.70(.59, .79)$ & $.89(.67, .89)$ & $.13(.00, .28)$ & $.60(.41, .76)$ & $.66(.53, .76)$ & $.82(.70, .90)$ \\
\hline Qol & $.64(.41, .38)$ & $.59(.35, .78)$ & $.32(.18, .46)$ & $.39(.13, .63)$ & $.57(.44, .67)$ & $.59(.37, .77)$ \\
\hline R & $.75^{* *}$ & $.85^{* *}$ & $.33^{* *}$ & $.66^{* *}$ & $.73^{* *}$ & $.87^{* *}$ \\
\hline$R^{2}$ & $.57^{* *}$ & $.74^{* *}$ & $.11^{* *}$ & $.42^{* *}$ & $.54^{* *}$ & $.75^{* *}$ \\
\hline
\end{tabular}

Abbrevations: K-SES Knee-Self Efficacy Scale, ACL Anterior Cruciate Ligament ACLR Anterior Cruciate Ligament Reconstruction, KOOS Knee Injury and Osteoarthritis Outcome Score, Pain KOOS pain, Sympt KOOS symptoms, ADL KOOS Activities of Daily Activities, Sport KOOS Sports and Leisure Activities, QOL KOOS quality of Life, $R$ multiple correlations; $R^{2}$ percentage of variation explained ${ }^{* *} p<.001$

developed to measure self-efficacy related to present daily activities, sports and leisure activities, and physical activities, are combined in the composite K-SES present $_{\text {scale. The }}$ meaning of the average K-SES present score is therefore not easy to interpret. As might be expected, K-SES- $\mathrm{D}_{\text {present }}$ is strongly correlated with self-reported functioning of the knee assessed with the KOOS, showing large overlaps in variation. The 4 items reflecting K-SES- $\mathrm{D}_{\text {future }}$ did not pose any problem in the CFA, and the scale score showed low correlations with current knee function. In addition, K-SES $S_{\text {future }}$ has been shown to be a better predictor of future functioning compared to K-SES present $_{\text {[13]. How- }}$ ever, as ACLR rehabilitation involves extensive exercises and training [4], the patients beliefs with regard to training and exercise are also important to the physical therapist.

This study is not without limitations. The selection of patients makes it hard to make direct comparisons between this study and outcomes reported in the original Swedish K-SES validation study. In the original study, ACL and ACLR patients were both used to determine different measurement properties [16]. However, although the samples of patients included in this study differ from the samples in the original study, average item scores in both factor obtained in the current study are similar to item scores reported earlier [13]. Furthermore, patients were not assessed at a fixed moment in time after ACL (sample 1) or ACLR (sample 2). It is unclear whether the reported differences in time since ACL or ACLR will impact these results. Another drawback of this study is that there are no data available on responsiveness of the K-SES-D. The original K-SES was shown to be sensitive to change as scores increased during rehabilitation after ACL tear [31]. Further research is needed to determine to what extent the K-SES-D is responsive to change. Finally, in this study data for test-retest reliability were assessed with an interval of 1 week. This interval was chosen to accommodate patients and therapists, and avoid additional visits to the physical therapist. However, COSMIN recommends an interval of 2 weeks to avoid recall of the items. Therefore, we do not know whether recall of items poses a problem in this study.

Despite these limitations, the study has important findings. The K-SES-D measures self-efficacy beliefs related to functioning in ACL and ACLR patients. Acceptability, internal consistency, and test-retest reliability of the subscales

Table 5 Correlation (95\% Confidence Interval) between K-SES-D subscales and self-report psychological variables

\begin{tabular}{lllr}
\hline & K-SES-D present & K-SES-D future & K-SES-D total \\
\hline TSK & $-0.66(-0.51,-0.78)$ & $-0.65(-0.46,-0.78)$ & $-0.74(-0.61,-0.83)$ \\
PCS & $-0.64(-0.40,-0.79)$ & $-0.54(-0.34,-0.72)$ & $-0.67(-0.42,-0.80)$ \\
MHLC Intern & $0.10(-0.16,0.40)$ & $-0.35(-0.11,-0.57)$ & $-0.19(-0.19,-0.48)$ \\
MHLC Extern & $0.32(0.09,0.57)$ & $0.34(0.11,0.55)$ & $0.36(0.10,0.59)$ \\
MHLC Physician & $0.15(-0.12,0.44)$ & $0.28(0.04,0.52)$ & $0.20(0.10,0.49)$ \\
HADS & $-0.29(-0.06,-0.56)$ & $-0.34(-0.04,-0.56)$ & $-0.32(-0.05,-0.37)$ \\
R & $0.74^{* *}$ & $0.71^{* *}$ & $0.80^{* *}$ \\
$R^{2}$ & $0.55^{* *}$ & $0.51^{* *}$ & $0.63^{* *}$ \\
\hline
\end{tabular}

Abbrevations: K-SES-D Knee Self Efficacy Dutch, TSK Tampa Scale Kinesophobia, PCS Pain Catastrophizing Scale, MHLC Multidimensional Health Locus of Control Scale, HADS Hospital Anxiety and Depression Scale, $R$ multiple correlations, $R^{2}$ percentage of variation explained ${ }^{* *} p<.001$ 
are good. Construct validity was confirmed in principal component analysis and hypothesis testing, but not in a confirmatory factor analysis. These self-efficacy beliefs are only weakly correlated to general self-efficacy beliefs. In ACLR patients, self-efficacy beliefs towards present functioning (in particular towards physical activity exercise) are different from self-efficacy beliefs about future functioning. Addressing these self-efficacy beliefs is important as most patients are motivated to return to previous levels of sports [32], and self-efficacy predicts future function [13]. Furthermore, self-efficacy is recognized as one of the main psychological factors associated with return to sports [33], and satisfaction with knee function after an ACLR [34]. A number of different available techniques to improve self-efficacy beliefs have been studied [35]. However, only one study reported on the effects of an intervention addressing self-efficacy in ACL patients [36]. This study concluded that a strategy to improve self-efficacy beliefs as part of a standard rehabilitation protocol did not result in a better outcome compared to a standard protocol. However, the study was limited in size, and no inclusion criteria related to self-efficacy were used. Interventions are likely to be more effective when addressing only those patients with low self-efficacy. In recent years, studies in ACL and ACLR have concluded that an individual, or tailored, approach is needed to increase return to sports [37]. Including only patients with low K-SES future in self-efficacy targeting interventions is likely to increase self-efficacy in these patients. Furthermore, other modes of interventions should be addressed as well. Cognitive behavioural approaches as described in the study of Thomee [36] heavily depends on social persuasion as a way to improve self-efficacy. Interventions using an exposure based approach within the fear-avoidance model should be considered as well [38]. Such interventions might also hold a stronger appeal for patients.

\section{Conclusions}

The translated Dutch version of the K-SES was acceptable for patients. Internal consistency and test-retest reliability of the K-SES-D subscales are satisfactory. The subscales measure self-efficacy related to present functioning $\left(\mathrm{K}-\mathrm{SES} \mathrm{S}_{\text {present }}\right)$, and self-efficacy beliefs related to knee function in the future (K-SES $\left.S_{\text {future }}\right)$. Construct validity of both subscales was confirmed by factor analysis and hypothesis testing. However, construct validity was not confirmed using a two model solution in CFA. Further research is needed to test responsiveness.

\section{Additional file}

Additional file 1: Figure S1. Samples used in the study. (DOCX $34 \mathrm{~kb})$

\section{Abbreviations}

ACL: Anterior Cruciate Ligament; ACLR: Anterior Cruciate Ligament Reconstruction; ADL: Activities of Daily Life; CFA: Confirmatory Factor Analysis; CFI: Comparative Fit Index; CMIN/DF: Chi-square corrected for Degrees of Freedom; COSMIN: Consensus based standard for the selection of health measurement instruments; FoH: Fear of Harm; HADS: Hospital Anxiety and Depression Scale; ICC: Intraclass Correlation Coefficient; KMO: Kaiser-Mayer-Olkin test; KOOS: Knee injury and Osteoarthritis Outcome Scale; K-SES: KNEE Self-Efficacy Scale; K-SESfuture: Knee self-efficacy related to future functioning; K-SES present: Knee self-efficacy related to present functioning; MHLC: Multidimensional Health Locus of Control; PCA: Principal Component Analysis; QOL: Quality of life; RMSE: Root Mean Square Error of Estimation; SEM: Standard Error of Measurement; SRMR: Standardized Root Mean square Residual; TSK: Tampa Scale of Kinesophobia

\section{Acknowledgments}

This study was possible with the help of the following students: A. van Kesteren, J. Roos, M. van Klaveren, M. van Til, C. van der Sluijs, Y Peeters, P. Rhebergen, A. Wassink.

\section{Funding}

Not applicable

\section{Availability of data and materials}

The datasets used and/or analysed during the current study are available from the corresponding author on reasonable request.

\section{Author's contributions}

WVL conceived of the study, and participated in its design, performed the statistical analysis, and coordination and drafted the manuscript. NvM, BH, YP included patients in the study, and did help to draft the manuscript. BS and RvC participated in the design of the study and helped draft the manuscript. All authors read and approved the final manuscript.

\section{Ethics approval and consent to participate}

Informed consent in writing was obtained from all participants in this study. Studies from which data have been included in the current study were approved by local medical ethics committees. The original study protocol for sample 1 was approved by the CMO Arnhem/Nijmegen (2013/368); and the protocol for studies involving sample 2 and 3 by the local ethics commission (ACPO 21.03/16) of the HAN University of Applied Sciences. The authors certify that they have no affiliation with or financial involvement in any organisation or entity with a direct financial interest in the subject matter or materials in the article.

\section{Consent for publication}

Not applicable.

\section{Competing interests}

The author(s) declare that they have no competing interest. No external funding was received for this work.

\section{Publisher's Note}

Springer Nature remains neutral with regard to jurisdictional claims in published maps and institutional affiliations.

\section{Author details}

${ }^{1}$ Research group Musculoskeletal Rehabilitation Nijmegen, HAN University of Applied Sciences, Kapittelweg, 33 Nijmegen, The Netherlands. ${ }^{2}$ Research Institute for Health Sciences, IQ healthcare, Radboud University Medical Center, Nijmegen, The Netherlands. ${ }^{3}$ Knee Expert Centre, Eindhoven, The Netherlands. ${ }^{4}$ Sport Medical Centre Papendal, Arnhem, The Netherlands. ${ }^{5}$ Rudolf Magnus Institute of Neurosciences, Department of Rehabilitation, Physical Therapy Science \& Sports, University Medical Center Utrecht, Utrecht, The Netherlands. ${ }^{6}$ Research Department, Kliniek ViaSana, Mill, The Netherlands. 
Received: 22 January 2018 Accepted: 21 February 2019

Published online: 07 March 2019

\section{References}

1. Sanders TL, Maradit Kremers H, Bryan AJ, Larson DR, Dahm DL, Levy BA, Stuart MJ, Krych AJ. Incidence of anterior cruciate ligament tears and reconstruction: a 21-year population-based study. Am J Sports Med. 2016;44(6):1502-7.

2. Thomeé R, Kvist J. Structured rehabilitation model with clinical outcomes after anterior cruciate ligament reconstruction. In: Sports Injuries: Prevention, Diagnosis, Treatment and Rehabilitation. Eds. Doral MN, Karlson J. Berlin: Springer; 2015. p. 1439-65.

3. Lobb $\mathrm{R}$, Tumilty $\mathrm{S}$, Claydon LS. A review of systematic reviews on anterior cruciate ligament reconstruction rehabilitation. Physical Therapy in Sport. 2012:13(4):270-8

4. van Melick N, van Cingel RE, Brooijmans F, Neeter C, van Tienen T, Hullegie W, Nijhuis-van der Sanden MW. Evidence-based clinical practice update: practice guidelines for anterior cruciate ligament rehabilitation based on a systematic review and multidisciplinary consensus. Br J Sports Med. 2016:50(24):1506-15.

5. Ardern CL, Osterberg A, Tagesson S, Gauffin H, Webster KE, Kvist J. The impact of psychological readiness to return to sport and recreational activities after anterior cruciate ligament reconstruction. Br J Sports Med. 2014:48(22):1613-9.

6. Kvist J, Ek A, Sporrstedt K, Good L. Fear of re-injury: a hindrance for returning to sports after anterior cruciate ligament reconstruction. Knee Surg Sports Traumatol Arthrosc. 2005;13(5):393-7.

7. Wierike S, Sluis A, Akker-Scheek I, Elferink-Gemser M, Visscher C. Psychosocial factors influencing the recovery of athletes with anterior cruciate ligament injury: a systematic review. Scand J Med Sci Sports. 2013;23(5):527-40.

8. Ardern CL, Taylor NF, Feller JA, Webster KE. A systematic review of the psychological factors associated with returning to sport following injury. Br J Sports Med. 2013:47(17):1120-6.

9. Everhart JS, Best TM, Flanigan DC. Psychological predictors of anterior cruciate ligament reconstruction outcomes: a systematic review. Knee Surg Sports Traumatol Arthrosc. 2015;23(3):752-62.

10. Ardern CL, Taylor NF, Feller JA, Whitehead TS, Webster KE. Psychological responses matter in returning to preinjury level of sport after anterior cruciate ligament reconstruction surgery. Am J Sports Med. 2013;41(7):1549-58.

11. Langford JL, Webster KE, Feller JA. A prospective longitudinal study to assess psychological changes following anterior cruciate ligament reconstruction surgery. Br J Sports Med. 2009:43(5):377-81.

12. Flanigan DC, Everhart JS, Glassman AH. Psychological factors affecting rehabilitation and outcomes following elective Orthopaedic surgery. J Am Acad Orthop Surg. 2015;23(9):563-70.

13. Thomeé $P$, Währborg $P$, Börjesson M, Thomeé R, Eriksson Bl, Karlsson J. Selfefficacy of knee function as a pre-operative predictor of outcome 1 year after anterior cruciate ligament reconstruction. Knee Surg Sports Traumatol Arthrosc. 2008;16(2):118-27.

14. Ardern $\mathrm{CL}$, Kvist J. What is the evidence to support a psychological component to rehabilitation programs after anterior cruciate ligament reconstruction? Current Orthopaedic Practice. 2016;27(3):263-8.

15. Bandura A. Guide for constructing self-efficacy scales. Self-efficacy beliefs of adolescents. 2006:5(307-337).

16. Thomeé $\mathrm{P}$, Währborg P, Börjesson M, Thomeé R, Eriksson Bl, Karlsson J. A new instrument for measuring self-efficacy in patients with an anterior cruciate ligament injury. Scand J Med Sci Sports. 2006;16(3):181-7.

17. Beaton DE, Bombardier C, Guillemin F, Ferraz MB. Guidelines for the process of cross-cultural adaptation of self-report measures. Spine. 2000;25(24):3186-91.

18. Mokkink LB, Terwee CB, Patrick DL, Alonso J, Stratford PW, Knol DL, Bouter LM, De Vet HC. The COSMIN checklist for assessing the methodological quality of studies on measurement properties of health status measurement instruments: an international Delphi study. Qual Life Res. 2010;19(4):539-49.

19. van Lankveld W, van Melick N, Habets B, Roelofsen E, Staal JB, van Cingel R. Measuring individual hierarchy of anxiety invoking sports related activities: development and validation of the photographic series of sports activities for anterior cruciate ligament reconstruction (PHOSA-ACLR). BMC Musculoskelet Disord. 2017;18(1):287.

20. Roos EM, Roos HP, Lohmander LS, Ekdahl C, Beynnon BD. Knee injury and osteoarthritis outcome score (KOOS) — development of a self-administered outcome measure. J Orthop Sports Phys Ther. 1998;28(2):88-96.
21. Miller RP, Kori SH, Todd DD. The Tampa Scale: a measure of kinisophobia. Clin J Pain. 1991:7(1):51.

22. Sullivan MJ, Bishop SR, Pivik J. The pain catastrophizing scale: development and validation. Psychol Assess. 1995:7(4):524.

23. Wallston KA. The validity of the multidimensional health locus of control scales. J Health Psychol. 2005;10(5):623-31.

24. Zigmond AS, Snaith RP. The hospital anxiety and depression scale. Acta Psychiatr Scand. 1983;67(6):361-70.

25. Mukaka M. A guide to appropriate use of correlation coefficient in medical research. Malawi Med J. 2012;24(3):69-71.

26. Williams B, Brown T, Onsman A. Exploratory factor analysis: a five-step guide for novices. Australasian Journal of Paramedicine. 2012;8(3):1.

27. Kline RB. Principles and practice of structural eqaution modeling. 3rd ed. New York: Guildford Press; 2010.

28. Cohen J. A power primer. Psychol Bull. 1992;112(1):155

29. Terwee CB, Mokkink LB, Knol DL, Ostelo RW, Bouter LM, de Vet HC. Rating the methodological quality in systematic reviews of studies on measurement properties: a scoring system for the COSMIN checklist. Qual Life Res. 2012;21(4):651-7.

30. Panariello RA, Stump TJ, Maddalone D. Postoperative rehabilitation and return to play after anterior cruciate ligament reconstruction. Operative Techniques in Sports Medicine. 2016;24(1):35-44

31. Thomeé $P$, Währborg P, Börjesson M, Thomeé R, Eriksson B, Karlsson J. Selfefficacy, symptoms and physical activity in patients with an anterior cruciate ligament injury: a prospective study. Scand J Med Sci Sports. 2007;17(3):238-45.

32. Sonesson S, Kvist J, Ardern C, Österberg A, Silbernagel KG. Psychological factors are important to return to pre-injury sport activity after anterior cruciate ligament reconstruction: expect and motivate to satisfy. Knee Surg Sports Traumatol Arthrosc. 2017;25(5):1375-84.

33. Senorski EH, Samuelsson K, Thomeé C, Beischer S, Karlsson J, Thomeé R. Return to knee-strenuous sport after anterior cruciate ligament reconstruction: a report from a rehabilitation outcome registry of patient characteristics. Knee Surg Sports Traumatol Arthrosc. 2017:25(5):1364-74.

34. Ardern CL, Österberg A, Sonesson S, Gauffin H, Webster KE, Kvist J. Satisfaction with knee function after primary anterior cruciate ligament reconstruction is associated with self-efficacy, quality of life, and returning to the preinjury physical activity. Arthroscopy. 2016;32(8):1631-1638.e3.

35. Wright BJ, O'Halloran PD, Stukas AA. Enhancing self-efficacy and performance: an experimental comparison of psychological techniques. Res Q Exerc Sport. 2016;87(1):36-46.

36. Thomeé $P$, Währborg P, Börjesson M, Thomeé R, Eriksson BI, Karlsson J. A randomized, controlled study of a rehabilitation model to improve kneefunction self-efficacy with ACL injury. J Sport Rehab. 2010;19(2):200-13.

37. Karlsson J, Becker R. Return to sports after $A C L$ reconstruction: individual considerations; 2015

38. Vlaeyen JW, Linton SJ. Fear-avoidance model of chronic musculoskeletal pain: 12 years on. Pain. 2012;153(6):1144-7.

Ready to submit your research? Choose BMC and benefit from:

- fast, convenient online submission

- thorough peer review by experienced researchers in your field

- rapid publication on acceptance

- support for research data, including large and complex data types

- gold Open Access which fosters wider collaboration and increased citations

- maximum visibility for your research: over $100 \mathrm{M}$ website views per year

At BMC, research is always in progress.

Learn more biomedcentral.com/submissions 